\title{
Elephants, mobility and captivity: what can these mighty and majestic animals teach us about joint health and osteoarthritis?
}

\author{
Ali Mobasheri ${ }^{1,2,3 *}$ and Carol Buckley ${ }^{4}$ \\ 1 Research Unit of Medical Imaging, Physics and Technology, Faculty of Medicine, University of Oulu, FI- \\ 90014, Oulu, Finland \\ 2 Department of Regenerative Medicine, State Research Institute Centre for Innovative Medicine, LT-08406, \\ Vilnius, Lithuania \\ 3 University Medical Center Utrecht, Departments of Orthopedics, Rheumatology and Clinical Immunology, \\ 3508 GA, Utrecht, The Netherlands \\ 4 Elephant Aid International, P.O. Box 283, Attapulgus, GA 39815, United States of America; \\ elevisions@gmail.com \\ * Correspondence: ali.mobasheri@oulu.fi
}

Simple Summary: African and Asian elephants are the largest animals that live on land. In order to find fresh food and water they need to be highly mobile and active. Despite their huge size, African elephants are nimble and can walk up to $28 \mathrm{~km}$ per day, although they usually walk $25 \mathrm{~km}$ on a daily basis. Asian elephants walk up to 13 miles each day and they can be active for up to 20 hours every day through walking, grazing, swimming and socializing. Mobility helps elephants maintain their joints and muscles. However, most captive-held elephants are kept in enclosures with hard surfaces (i.e. concrete, tarmac and hard backed dirt), often in very small spaces. Therefore, captiveheld elephants cannot exercise as much as wild-living elephants. This makes them more prone to gaining weight, becoming obese, lame and developing bone and joint diseases such as arthritis, especially osteoarthritis (OA). It has been estimated that $50 \%$ of captive-held elephant deaths are caused by the lack of mobility. This perspective article focuses on the link between mobility, captivity and the development of OA in elephants.

\begin{abstract}
The African bush and forest elephants, Loxodonta Africana and Loxodonta cyclotis, and the Asian elephant, Elephas maximus, are the largest land-dwelling animals. Elephants need to be highly mobile and active in order to find fresh food and water, and in the case of males, to locate females in estrus for breeding. Asian elephants walk up to 13 miles each day and African elephants can walk up to $28 \mathrm{~km}$ per day. This high level of mobility in the wild is also important for maintaining an optimum musculoskeletal health. However, their captive counterparts live in restricted spaces and cold climates that require extended periods of indoor confinement are therefore unable to be as physically active. Zoo enclosures for elephants are relatively small with hard surfaces (i.e. concrete, tarmac and hard packed dirt), so they cannot exercise and are forced to stand on unnaturally hard surfaces continually. Physical inactivity in captivity makes them more prone to gaining weight and developing bone and joint diseases such as osteomyelitis, joint ankylosis and osteoarthritis (OA). Up to $50 \%$ of deaths in captive elephants are caused by the lack of mobility. This perspective article focuses on the link between captivity, mobility, physical inactivity and the development of OA in captive elephants.
\end{abstract}

Keywords: osteoarthritis; African elephant; Asian elephant; captivity; mobility

\section{Introduction}

Osteoarthritis (OA) is the most common form of arthritis in the world, the leading cause of disability and the primary source of societal cost in older adults [1]. According to the World Health 
Organization (WHO), OA affects millions of people worldwide ${ }^{1}$. Recent studies suggest that OA affects $7 \%$ of the global population, which is more than 500 million people worldwide, with women disproportionately affected by the condition [2], especially after menopause [3-5]. Although OA is primarily related to aging, it is, along with many other forms of chronic disease, also associated with a wide variety of modifiable and non-modifiable risk factors that include: overweight and obesity [6,7], sedentary behavior [8] and lack of physical exercise [9]. In addition to the primary risk factors of aging, obesity, gender, and genetics, other inciting risk factors for OA may include previous joint trauma or history of repetitive joint injuries or even the presence of metabolic syndrome and endocrine disease [10]. However, the disease is primarily biomechanical. There are biomechanical [11], inflammatory [12], and metabolic [13] factors that have been demonstrated to play dominant roles in the initiation and progression of OA.

\section{Mobility and the evolution and migration of modern Homo sapiens}

Mobility was essential for the evolution of Homo sapiens and an essential component of the "Global Human Journey" [14,15]. Humans evolved from an ancestor that was not limited to trees or other elevated habitats. Our human ancestors were highly mobile and agile, which was essential for gathering food and finding new shelter [16]. The ability to move and migrate allowed them to colonize the entire planet and settle on every continent [17]. Now that we have colonized and dominated every corner of the globe we face a new set of challenges. The rising global burden musculoskeletal (MSK) diseases is now threatening one of the key the human qualities that allowed us to become the dominant species: mobility. The recent Global Burden of Disease (GBD) Study estimated the burden disability in 187 countries and 21 regions of the world for the years 1990, 2010 and 2013 of all MSK disorders. OA rheumatoid arthritis (RA), gout, low back pain (LBP), neck pain (NP) and all other MSK disorders combined caused $21.3 \%$ of the total years lived with disability (YLDs) globally $[18,19]$. MSK health is critical for human function, enabling mobility, dexterity and the ability to work and actively participate in all aspects of life [20]. Global MSK health targets should also be set to reflect maintenance of mobility, participation and physical function as key components of functional ability and performance [21].

\section{What can we learn about joint health and OA from the African elephant Loxodonta Africana and the Asian elephant Elephas maximus?}

We design and conduct clinical trials to look at the effects of physical activity and inactivity on joint health and OA, focusing on humans as the primary target population [22]. However, there is a great deal that we can learn from large animals. Elephants are the largest land-dwelling mammal. They have evolved a specialized foot morphology to help reduce locomotor pressures while supporting their large body mass [23]. Elephant limbs display unique morphological features which are related mainly to supporting their enormous body weight and the knee joint plays crucially important roles in weight bearing and locomotion [24]. They are not perhaps the largest land dwelling mammals that roamed the Earth; the woolly mammoth (Mammuthus primigenius), Deinotherium and Palaeoloxodon namadicus were the largest mammals to walk on the planet. Elephants and their ancestors were designed to be highly mobile mammals. Wild modern elephants need to be highly agile to find fresh food and water, and this mobility helps them maintain optimal musculoskeletal health. Despite their massive size, African elephants are nimble and can walk up to $28 \mathrm{~km}$ per day. Asian elephants walk up to 13 miles each day and they can be active for up to 20 hours every day through walking, grazing, swimming and socializing. African elephants have the capacity to walk up to 50 miles every day, but they mostly spend their day by walking near a water source so they can rehydrate themselves. However, recent research suggests that captive elephants can only walk up to a maximum of $5 \mathrm{~km}$ per day [25]. Furthermore, in zoos elephants are kept in enclosures with hard surfaces (i.e. concrete, tarmac and hard packed dirt) and small spaces and they cannot

\footnotetext{
${ }^{1}$ https://www.who.int/medicines/areas/priority_medicines/Ch6_12Osteo.pdf
} 
exercise as much as wild animals [26]. This raises important questions for the management of these animals, highlighting the crucially important areas of stress, obesity and poor adult survivorship among captive elephants [26]. Captivity makes them more prone to gaining weight and developing diseases such as osteomyelitis, joint ankylosis and OA. It is estimated that $50 \%$ of captive elephant deaths are caused by lack of mobility [27]. Therefore, elephants can teach us a great deal about the link between mobility, captivity and the development of OA associated with sedentary behavior. In fact, it could be argued that the captive elephant is a perfect large animal model for the studying the link between sedentarism, lack of mobility and OA.
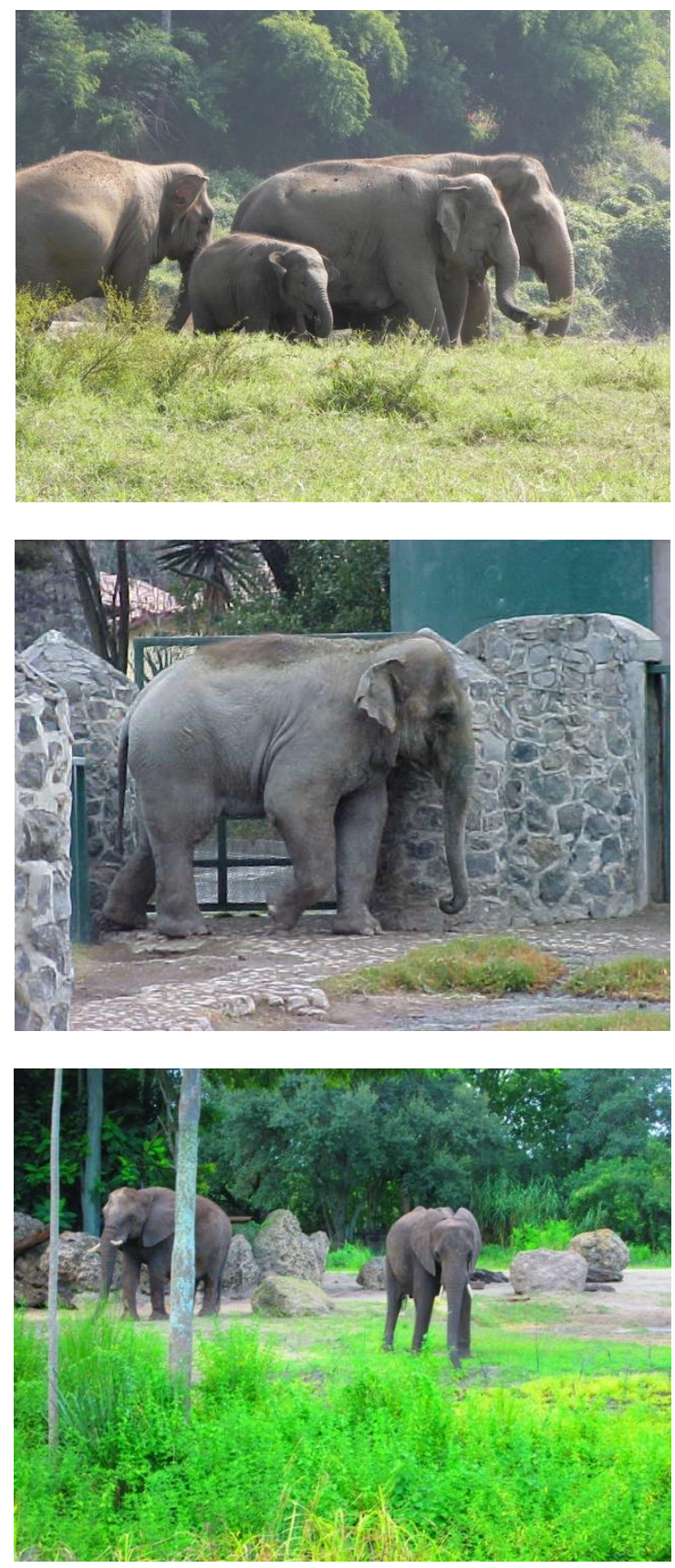

Figure 1. Asian elephants in the wild (top panel) and in captivity with limited space (middle panel). African elephants with more space in a reserve (bottom panel).

In humans obesity and lack of physical activity are major contributors to the development of OA [28]. However, there is no convincing published evidence in humans that walking and running contributes to OA [29][30]. Therefore, it is hardly surprising that captive elephants suffer from lameness and OA as they have to stand for long periods of time on hard surfaces and they are unable 
to exercise enough and walk the normal distances that they would in the wild every day. They cannot be active in very cold climates and in small zoo enclosures where their freedom and mobility is highly restricted. These are important ethical and welfare considerations that must be taken into account in future captivity and conservation programs.

There are currently no effective drugs and pharmacological treatments for OA [31]. Recent clinical research suggests that weight loss, physical activity and increasing muscle mass and strength are the only effective strategies for reducing pain and enhancing mobility in subjects with OA [32]. The only way to slow down the pain and progression of OA appears to be physical exercise, avoiding obesity and maintaining a healthy weight [33-36]. Non-steroidal anti-inflammatory drugs (NSAIDs) can only treat the symptoms of OA. Studies have examined the pharmacokinetics of orally administered phenylbutazone in African and Asian elephants and the evidence suggests that different treatment regimens should be used for each species, based on size and weight [37]. Similar studies have established the optimal dosage for using ketoprofen [38] and ibuprofen [39] in elephants. However, these NSAIDs can be highly toxic to the gastrointestinal tract in humans and in large animals such as horses and elephants, causing general toxicity, colitis and inhibition of mucosal barrier healing [40]. Therefore, the only strategy currently available to slow OA progression in elephants is to allow them to exercise and walk and look after their feet by limiting walking on unnaturally hard surfaces. There is no solid research in this area and most of the evidence is anecdotal and provided by wildlife and zoo veterinarians. Meanwhile, OA researchers are looking for new therapies for this disease in humans and non-human animal models and all the evidence from captive elephants suggests that captivity and reduced physical activity can accelerate the development of OA. Therefore, the physical activity that has been recommended for human patients with OA can also be recommended for elephants, who need it as much as we do.

\section{Conclusions}

Physical inactivity and sedentary behavior contribute to poor health in humans. Captivity and reduced mobility are also major contributors to poor health in captive animals, including elephants. Wild elephants are highly mobile and require the freedom and space to reach their daily activity goals, often called "lumbering". Elephants in small enclosures in zoos do not get enough exercise compared to their counterparts in the wild. Elephants in Safari parks and sanctuaries may have more freedom to move around but unless they live in reserves and national parks, they do not have enough space and opportunities for "lumbering" or "hurtling" the long distances that they were evolutionary designed for. Captive elephants have a shorter lifespan. Keeping them in captivity may protect them from poaching but the protection afforded to them presents a whole new set of health challenges, including overweight, obesity, lameness and OA. Several years ago the American Zoological Association (AZA) published research proposing that captive Asians live on average 44 years. They also claimed wild Asian elephants live about the same length of time. However, from our experience there is a problem with this claim pertaining to wild elephants. There are no long-term studies of wild Asian elephants to support such a claim. However, there is much information about captive elephants living into their 80's all over Asia. The reason the captive elephants in Asia live much longer than the captive elephants in the Western world is due to climate and management. In Asia, until very recently, captive elephants lived in the jungles and worked in the lumber industry. They did not live on hard surfaces, they were fed a natural diet, they got plenty of exercise and usually were with conspecifics. More recently, as the captive-held elephant in Asia has been brought out of the jungle and into cities for tourist entertainment, their health has suffered, disease is prevalent, and lives are shorter. In conclusion, elephants confined in small spaces without adequate exercise, living on unnaturally hard surfaces suffer from musculoskeletal disease and pain and consequently die early.

Author Contributions: Conceptualization, A.M., C.B.; writing-original draft preparation, A.M.; writingreview and editing, A.M., C.B.

Funding: A.M. has received funding from the following sources: The European Commission Framework 7 program (EU FP7; HEALTH.2012.2.4.5-2, project number 305815; Novel Diagnostics and Biomarkers for Early 
Identification of Chronic Inflammatory Joint Diseases). The Innovative Medicines Initiative Joint Undertaking under grant agreement No. 115770, resources of which are composed of financial contribution from the European Union's Seventh Framework program (FP7/2007-2013) and EFPIA companies' in-kind contribution. A.M. also wishes to acknowledge funding from the European Commission through a Marie Curie IntraEuropean Fellowship for Career Development grant (project number 625746; acronym: CHONDRION; FP7PEOPLE-2013-IEF). A.M. also wishes to acknowledge financial support from the European Structural and Social Funds (ES Struktūrinès Paramos) through the Research Council of Lithuania (Lietuvos Mokslo Taryba) according to the activity "Improvement of researchers" qualification by implementing world-class R\&D projects' of Measure No. 09.3.3-LMT-K-712 (grant application code: 09.3.3-LMT-K-712-01-0157, agreement No. DOTSUT215) and the new funding program: Attracting Foreign Researchers for Research Implementation (2018-2022).

Acknowledgments: A.M. would like to acknowledge members of his research teams and collaborators for their support and encouragement.

Conflicts of Interest: C.B. is the founder of Elephant Aid International, a charitable organization dedicated to the health and welfare of elephants worldwide. https://elephantaidinternational.org. A.M. is a member of the Integrative Health Team of Elephant Aid International. https:/elephantaidinternational.org/about/integrativehealth-team/. The authors declare no conflicts. The funders had no role in the decision to publish this paper.

\section{References}

1. Hunter, D. J.; Bierma-Zeinstra, S. Osteoarthritis. Lancet 2019, 393, 1745-1759.

2. Hunter, D. J.; March, L.; Chew, M. Osteoarthritis in 2020 and beyond: a Lancet Commission. Lancet 2020, 396, $1711-1712$

3. Hanna, F. S.; Wluka, A. E.; Bell, R. J.; Davis, S. R.; Cicuttini, F. M. Osteoarthritis and the postmenopausal woman: Epidemiological, magnetic resonance imaging, and radiological findings. Semin. Arthritis Rheum. 2004, $34,631-636$.

4. Karsdal, M. A.; Bay-Jensen, A. C.; Henriksen, K.; Christiansen, C. The pathogenesis of osteoarthritis involves bone, cartilage and synovial inflammation: may estrogen be a magic bullet? Menopause Int 2012, 18, 139-146.

5. Stevenson, J. C. A woman's journey through the reproductive, transitional and postmenopausal periods of life: impact on cardiovascular and musculo-skeletal risk and the role of estrogen replacement. Maturitas 2011, 70, 197-205.

6. Felson, D. T. Weight and osteoarthritis. Am. J. Clin. Nutr. 1996, 63, 430S-432S.

7. Zhang, Y.; Jordan, J. M. Epidemiology of osteoarthritis. Clin Geriatr Med 2010, 26, 355-369.

8. Blüher, M. Obesity: global epidemiology and pathogenesis. Nat. Rev. Endocrinol. 2019, 15, 288-298.

9. Booth, F. W.; Roberts, C. K.; Laye, M. J. Lack of exercise is a major cause of chronic diseases. Compr. Physiol. 2012, 2, 1143-1211.

10. Felson, D. T. Risk factors for osteoarthritis: understanding joint vulnerability. Clin. Orthop. Relat. Res. 2004, S16-21.

11. Englund, M. The role of biomechanics in the initiation and progression of OA of the knee. Best Pract Res Clin Rheumatol 2010, 24, 39-46.

12. Berenbaum, F. Osteoarthritis as an inflammatory disease (osteoarthritis is not osteoarthrosis!). Osteoarthr. Cartil. 2013, 21, 16-21.

13. Mobasheri, A.; Rayman, M. P.; Gualillo, O.; Sellam, J.; van der Kraan, P.; Fearon, U. The role of metabolism in the pathogenesis of osteoarthritis. Nat. Rev. Rheumatol. 2017, 13, 302-311.

14. Stringer, C.; McKie, R. African exodus: The origins of modern humanity. 1998.

15. Harari, Y. N. Sapiens: A brief history of humankind. 2014.

16. Lieberman, D. E. The story of the human body: evolution, health and disease. Fam Med 2016, 48, 822-823.

17. Bellwood, P. First migrants: ancient migration in global perspective. 2014.

18. Smith, E.; Hoy, D. G.; Cross, M.; Vos, T.; Naghavi, M.; Buchbinder, R.; Woolf, A. D.; March, L. The global 
burden of other musculoskeletal disorders: estimates from the Global Burden of Disease 2010 study. Ann. Rheum. Dis. 2014, 73, 1462-1469.

19. Woolf, A. D. Global burden of osteoarthritis and musculoskeletal diseases. BMC Musculoskelet. Disord. 2015, 16.

20. Briggs, A. M.; Cross, M. J.; Hoy, D. G.; Sànchez-Riera, L.; Blyth, F. M.; Woolf, A. D.; March, L. Musculoskeletal health conditions represent a global threat to healthy aging: A report for the 2015 world health organization world report on ageing and health. Gerontologist 2016, 56 Suppl 2, S243-55.

21. Milte, R.; Crotty, M. Musculoskeletal health, frailty and functional decline. Best Pract Res Clin Rheumatol 2014, 28, 395-410.

22. McAlindon, T. E.; Driban, J. B.; Henrotin, Y.; Hunter, D. J.; Jiang, G. L.; Skou, S. T.; Wang, S.; Schnitzer, T. OARSI Clinical Trials Recommendations: Design, conduct, and reporting of clinical trials for knee osteoarthritis. Osteoarthr. Cartil. 2015, 23, 747-760.

23. Panagiotopoulou, O.; Pataky, T. C.; Day, M.; Hensman, M. C.; Hensman, S.; Hutchinson, J. R.; Clemente, C. J. Foot pressure distributions during walking in African elephants (Loxodonta africana). R. Soc. Open Sci. 2016, $3,160203$.

24. Weissengruber, G. E.; Fuss, F. K.; Egger, G.; Stanek, G.; Hittmair, K. M.; Forstenpointner, G. The elephant knee joint: morphological and biomechanical considerations. J. Anat. 2006, 208, 59-72.

25. Holdgate, M. R.; Meehan, C. L.; Hogan, J. N.; Miller, L. J.; Soltis, J.; Andrews, J.; Shepherdson, D. J. Walking Behavior of Zoo Elephants: Associations between GPS-Measured Daily Walking Distances and Environmental Factors, Social Factors, and Welfare Indicators. PLoS One 2016, 11, e0150331.

26. Clubb, R.; Rowcliffe, M.; Lee, P.; Mar, K. U.; Moss, C.; Mason, G. J. Compromised survivorship in zoo elephants. Science 2008, 322, 1649.

27. Bansiddhi, P.; Brown, J. L.; Thitaram, C. Welfare assessment and activities of captive elephants in thailand. Animals (Basel) 2020, 10.

28. Felson, D. T.; Anderson, J. J.; Naimark, A.; Walker, A. M.; Meenan, R. F. Obesity and knee osteoarthritis. The Framingham Study. Ann. Intern. Med. 1988, 109, 18-24.

29. Eichner, E. R. Does running cause osteoarthritis? Phys. Sportsmed. 1989, 17, 147-154.

30. Williams, P. T. Effects of running and walking on osteoarthritis and hip replacement risk. Med. Sci. Sports Exerc. 2013, 45, 1292-1297.

31. Ghouri, A.; Conaghan, P. G. Update on novel pharmacological therapies for osteoarthritis. Ther Adv Musculoskelet Dis 2019, 11, 1759720X19864492.

32. Messier, S. P.; Loeser, R. F.; Miller, G. D.; Morgan, T. M.; Rejeski, W. J.; Sevick, M. A.; Ettinger, W. H.; Pahor, M.; Williamson, J. D. Exercise and dietary weight loss in overweight and obese older adults with knee osteoarthritis: the arthritis, diet, and activity promotion trial. Arthritis Rheum. 2004, 50, 1501-1510.

33. Bennell, K.; Hinman, R. Exercise as a treatment for osteoarthritis. Curr. Opin. Rheumatol. 2005, 17, 634-640.

34. Bliddal, H.; Leeds, A. R.; Christensen, R. Osteoarthritis, obesity and weight loss: evidence, hypotheses and horizons - a scoping review. Obes. Rev. 2014, 15, 578-586.

35. Yu, S. P.; Hunter, D. J. Managing osteoarthritis. Aust. Prescr. 2015, 38, 115-119.

36. Barrow, D. R.; Abbate, L. M.; Paquette, M. R.; Driban, J. B.; Vincent, H. K.; Newman, C.; Messier, S. P.; Ambrose, K. R.; Shultz, S. P. Exercise prescription for weight management in obese adults at risk for osteoarthritis: synthesis from a systematic review. BMC Musculoskelet. Disord. 2019, 20, 610.

37. Bechert, U.; Christensen, J. M.; Nguyen, C.; Neelkant, R.; Bendas, E. Pharmacokinetics of orally administered 
phenylbutazone in African and Asian elephants (Loxodonta africana and Elephas maximus). J. Zoo Wildl. Med. 2008, 39, 188-200.

38. Hunter, R. P.; Isaza, R.; Koch, D. E. Oral bioavailability and pharmacokinetic characteristics of ketoprofen enantiomers after oral and intravenous administration in Asian elephants (Elephas maximus). Am J Vet Res 2003, 64, 109-114.

39. Bechert, U.; Christensen, J. M. Pharmacokinetics of orally administered ibuprofen in African and Asian elephants (Loxodonta africana and Elephas maximus). J. Zoo Wildl. Med. 2007, 38, 258-268.

40. Marshall, J. F.; Blikslager, A. T. The effect of nonsteroidal anti-inflammatory drugs on the equine intestine. Equine Vet. J. Suppl. 2011, 140-144. 